\title{
Role of community pharmacist in management of anaemia
}

\begin{abstract}
Anaemia is a condition in which number of RBC cells are decreased or oxygen carrying capacity of blood is reduced, i.e enough haemoglobin is not available to carry sufficient oxygen from the lungs to supply the needs of the tissues. Iron is an important essential element for almost all living organisms in various metabolic processes, including oxygen transport, synthesis of deoxyribonucleic acid (DNA), electron transport etc. Haematinics are the drugs which are commonly prescribed in the initial treatment of anaemia and also given during pregnancy and lactation phase. Various types of oral iron preparations(Tablets, syrups, capsules) are available in the market as single or fixed dose combination with other minerals, micronutrients etc. Bioavailability of oral iron preparations depends upon the type of dosage form, its dose, age, sex, food and liquids that are taken along with the iron supplements. Presence of minerals or vitamins in combination also influences the absorption of iron. The main objective of present paper is to elaborate the role of pharmacist in improving bioavailability of haematinics by educating the consumer in selecting right dosage form, right combination and right strength.
\end{abstract}

Keywords: anaemia, iron supplements, Microcytic anaemia, community pharmacist
Volume 6 Issue 3 - 2018

Ramu B, Saibaba SV

Department of Pharmacy practice, KVK College of Pharmacy, India

Correspondence: Ramu B, Department of Pharmacy practice, KVK College of Pharmacy, India,

Email bandameedi.ramu@gmail.com

Received: May 15, 2018 | Published: June 042018

\section{Introduction}

The word anemia derived from Greek words 'an' means no and 'naemia' means blood. Anaemia is defined as a condition where the total number of Red blood cells is decreased and their oxygen carrying capacity is reduced. The normal value of Haemoglobin in men is 13$14 \mathrm{gm} / 100 \mathrm{ml} \&$ in women $12 \mathrm{gm} / 100 \mathrm{ml}$ of blood. If men \& women having haemoglobin less than the normal values are considered as anaemic. ${ }^{1-4}$

\section{Normal daily recommended intake (in $\mathrm{mg}$ )}

Infant's upto3yrs: 6-10mg.

\section{4-10yrs: $10 \mathrm{mg}$}

Pregnant women: $30 \mathrm{mg}$.

Breast feeding females: $15 \mathrm{mg}$.

Anaemia is most common in Females, Children and Elderly.

The symptoms of anaemia include headache, pale skin and tongue, pale eyes \& nails, dizziness. Short breath, fatigue etc.

Anaemia are of several types: ${ }^{5-7}$

a. Macrocytic or Megaloblastic Anaemia

b. Microcytic Anaemia

c. Haemolytic Anaemia

d. Aplastic Anaemia

e. Thalassaemia

f. Sickle cell Anaemia

g. Normocytic Anaemia.

h. Pernicious anaemia

\section{Macrocytic or megaloblastic anemia}

This anemia is due to deficiency of vitamin $\mathrm{B}_{12}$ or folic acid results in abnormally large erythrocytytes (megaloblasts). The rate of DNA \& RNA systhesis is reduced, delaying cell division. Therefore, the cells can grow larger than the normal between divisions. Vitamin $\mathrm{B}_{12}$ and folic acid play an essential role in the production of red blood cells.

\section{Causes}

i. It occurs most often in females than males usually between 45 to $65 \mathrm{yrs}$ of age. This is an auto immune disease in which auto antibodies destroy intrinsic factors \& parietal cells.

ii. Chronic gastritis, Malignant diseases, Ionizing radiation-damage the gastric mucosa including parietal cells that produce intrinsic factor.

iii. Patients who have intestinal sprue, folic acid, vitamin $B_{12}$ \& other vitamin $\mathrm{B}$ complex compounds are poorly absorbed. These vitamins play an important role in the production of red blood cells.

iv. Other causes include haemolysis, hemorrhage, cirrhosis, protein deficiency, scurvy, alcohol ingestion, bone marrow infiltration or replacement etc.

\section{Microcytic anaemia or Hypochromic anaemia/iron deficiency anaemia}

This anaemia is regarded as severe, when the haemoglobin level is below $9 \mathrm{gm} / \mathrm{dl}$ of blood. It is caused by deficiency of iron in the bone marrow and may be due to dietary deficiency or mal-absorption (No absorption). This is one of the most common forms of anaemia that affects nearly one billion people worldwide. In India $50 \%$ of teenage girls, $60 \%$ of pregnant women $\& 80 \%$ of children (below5yrs) are found to be anaemic according to Third National family Health survey which is conducted in 2005-06. In this type of anaemia erythrocytes are small (microcytes) and pale than normal (Hypochromic) as their 
haemoglobin content is low. Iron deficiency occurs when the rate of use or loss of iron is more than its use or rate of absorption.

\section{Causes}

a. Chronic blood loss: This is due to chronic peptic ulcers, menorrhage (due to menstrual cycle) intestinal ulceration, haemorroids (piles) carcinoma, uremia etc.

b. Reduced absorption of Iron: This is due to chronic diarrhea, malabsorption, achlorhydria or hypochlorhydria, partial surgical removal of stomach etc.

c. Excessive use of iron: There is an increased demand of iron during pregnancy because of growth of fetus and for the children who are in infant stage \& Adolescence stage require extra iron. If this is not provided through supplements, iron deficiency anaemia occurs in. The normal daily requirements of iron intake for men are about 1-2 $\mathrm{mg}$, for a woman is $3 \mathrm{mg}$ and for children during their period of rapid growth require more than adults.

\section{Signs and symptoms}

The common symptoms include weakness, short breath, tiredness, swelling at corners of the mouth, tongue becomes pale \& inflamed.

\section{Haemolytic anaemia}

If $\mathrm{RBC}$ plasma membranes rupture prematurely, the cells remain as ghosts and their haemoglobin pours out into the plasma. This condition is called haemolytic anaemia. In these diseases, genetic abnormality leads to synthesis of abnormal haemoglobin \& increased red blood cell membrane friability, reducing cell oxygen carrying capacity and life span. Haemolytic anaemia also caused by parasites, toxins and antibodies from incompatible blood. This type of anaemia can be treated by surgery, blood transfusion, medicines, lifestyle changes and bone marrow transplantation. The common form of haemolytic anaemia is Sickle cell anaemia and Thalassaemia.

\section{Sickle cell anaemia}

Sickle cell anaemia is a lifelong, serious disease in which the abnormal haemoglobin molecules become misshapen when deoxygenated and makes the RBC sickle ('C' shaped) shaped. Normal RBC are disc shaped and easily move through blood vessels. But sickle cells contain abnormal haemoglobin that causes cells to have sickle shape which cannot move smoothly through blood vessels. A high production of abnormal molecules makes the sickling permanent. The life span of cells is reduced to early haemolysis. These sickle cells do not move smoothly through blood vessels and tend to increase the viscosity of blood, reducing the rate of blood flow $\&$ leading to intravascular clotting, ischemia and infarction. Such patients frequently go into vicious circle called a sickle cell disease. Crisis in which low oxygen tension in the tissue causes sickling which causes ruptured red cells, thus in turn causing still further damage in oxygen tension and still more sickling \& red cell destruction. Once the process starts, it progress rapidly leading to serious decrease in RBC cell mass within a few hours and often death. Bone marrow transplants can cure sickle cell anaemia in initial stages.

\section{Thalassaemia}

Thalassaemia represents a group of hereditary haemolytic anaemia resulting from a defect in synthesis of haemoglobin which produces extremely thin and fragile RBC cells. Severe cases may cause death in infants or young children. It occurs primarily in population from countries bordering the Mediterranean Sea.

\section{Causes}

Paracytic diseases eg: malaria. Ionizing radiation: X-rays, Radioactive isotopes, destruction of blood trapped in tissues, eg: severe burns, crushing injuries, physical damage to cells eg: by artificial heart valves, kidney dialysis machine etc. The treatment for Thalassaemia is blood transfusion.

\section{Aplastic anaemia}

Bone marrow aplasia means lack of functioning of bone marrow. Aplastic anaemia is usually accomplished by abnormal leukocyte and thrombocyte production and this condition is called pancytopenia. Aplastic anaemia is rare but serious condition. It can develop slowly or suddenly. The condition is often idiopathic and the causes include:

a) Drugs: cytotoxic drugs, some anti-convulsant drugs, some sulphonamides \& antibiotics.

b) Some chemicals like Benzene and its derivatives

c) Virus diseases including hepatitis.

d) Ionizing radiation i.e., A person exposed to gamma rays radiation from a nuclear bomb blast is likely to sustain complete destruction of bone marrow. Likewise, excessive X-ray treatment certain industrial chemicals etc.

\section{Normocytic anaemia}

In this, the cells are normal but the numbers are reduced and the proportion of reticulocytes in the blood may be increased. This occurs in many chronic disease conditions like chronic inflammation, haemolytic diseases etc. Common causes are large wounds, stomach ulcers, heavy menstrual bleeding.

\section{Treatment anaemia}

Balanced Diet: Anaemia can be prevented by balanced dietary supplements. Foods that are iron rich help in maintaining haemoglobin levels if they consumed daily in proper proportion. There are two types of iron rich dietary,1) Haem Iron 2) Non-haem Iron. Haem iron comes from animal source foods and non-haem iron comes from plant based and animal-based foods. Haem iron is easily and more uniformly absorbed (15-35\%) than non-haem iron which has poor and variable bioavailability (5-12\%). Vegetarian foods like legumes, cereals, beans, pulses, whole grains and leafy vegetables contain equal amount of iron which equivalent to amounts in non-vegetarian foods. The iron in vegetable food is less available for absorption due to differences in chemical form of iron (3) and the accompanying components that enhance or inhibit iron absorption (3) People of India are mostly vegetarians therefore they become vulnerable to anaemia. Wide variety of iron supplements is available in the market. Solid oral products constitute almost $55-60 \%$ of total haematinic market followed by liquid preparations $30-35 \%$ followed by parenteral up to $4-5 \%$. Oral supplements are most commonly prescribed as they are cheap ,safe and effective \& also because of increasing haemoglobin levels, maintaining proper iron stores to prevent and correct iron deficiencies.

The recommended therapy for the treatment of anaemia is 60 $200 \mathrm{mg}$ of iron in 2-3 divided doses for three month, because as the amount of iron absorbed decrease with dosage increase, taking 2-3 equally spaced doses per day generally recommended. Absorption 
is high when given in empty stomach. But the patient will suffer sometimes with nausea and epigastric pain. Intravenous iron as a total dose infusion is able to replenish iron stores more efficiently, completely and at a faster rate than oral therapy which provides fuel for stimulation of full erythropoesis compared to oral iron. Selection of a particular drug or combination with other nutrients shows a significant influence on bioavailability of iron. The absorption of iron not only depends on type of dosage form but also on the patients with iron deficiency condition. Wide variety of iron preparations are available in the market in different formulations including capsules, tablets, powders, granules, syrups, drops, injections etc. Iron products are also available in immediate release dosage forms and extended release dosage form. Therefore it is really confuses the prescriber as well as consumer to select right iron supplements, especially when there is so much of variation in formulation release pattern mechanism, salt forms etc. Apart from being available in a single supplement form, combination of iron with vitamins, minerals, amino acids are available which further confuses the doctor and patient alike. Therefore, community pharmacist who dispenses these dosage forms should play an important role in the usage and educating the consumer about the efficacy of these products.

WHO model list of essential medicines recommends a ferrous salt form rather than Ferric state form to treat anaemia since ferrous salts have better bioavailability $(10-15 \%)$ than ferric form $(5 \%)$. Ferric salts are having three to four (3-4) times lesser bioavailability compared to ferrous salts due to Poor solubility of ferric salt in intestinal fluids and also because of the fact that ferric form needs to be transformed into ferrous form before being absorbed. The most popular iron (III) hydroxide polymaltose complex is a chelate that has pleasant taste and does not cause gastrointestinal irritation. But most of clinical studies have shown that it has poor bioavailability and high cost of this preparation restricted its use. Among ferrous formulations, ferrous sulphate remains popular and is used for treatment of anaemia. It is highly effective with acceptable tolerability and low cost. Clinical trials of other ferrous salts like ferrous fumerate, ferrous citrate, ferrous succinate, and ferrous gluconate has shown comparable bioavailability with that of ferrous sulphate formulations. ${ }^{8-12}$

Indian pharmaceutical markets are flooded with preparations of combination of iron with minerals, vitamins, amino acids and other micronutrients. Haematopoiesis require excess supply of minerals, for eg: iron, copper, and vitamins like vitamin $\mathrm{B}_{12}$, folic acid, Riboflavin, vitamin $\mathrm{B}_{6}$, and various haematopoietic growth factors. Each element is effective individually in particular type of anemia but because of unnecessary and wrong use of these drugs frequently, without determining their applicability, the things got worse. Many of these dosage forms contain several ingredients that don't have nothing to do with hematopoiesis. For eg: why a pernicious anemia patient should be treated with iron, ascorbic acid or folic acid. He requires vitamin $\mathrm{B}_{12}$. Preparations containing less than $33 \mathrm{mg}$ of iron require more than three doses per day to make up therapeutic dose of $100 \mathrm{mg}$ per day which can adversely affect the patient. It has been clinically proved that combination of iron and calcium or iron and zinc in a supplement leads to poor absorption of iron and prolonged use of such supplements doesn't increase hemoglobin content. Therefore such supplements should be avoided. Combination of iron with folic acid and iron with ascorbic can be considered as rational combination because ascorbic acid improves absorption of iron by reducing ferric form to ferrous form. Anemia (macrocytic anemia) due to deficiency of folic acid is quite common hence a combination therapy with iron is most acceptable. ${ }^{13-14}$

\section{Modified (immediate or extended) release formulations}

These formulations are designed in such way that it releases iron gradually. As the preparation enters into gastrointestinal tract at any given time, only a small amount of iron is present in the lumen. The main advantage of these formulations is less GI irritation and long onset action limiting the dose to once or twice daily. There is controversy on the bioavailability of these formulations that few clinical studies report better bioavailability with fewer side effects while other shows no advantage.

\section{Cost of iron supplements}

Formulations of iron dextran injection, iron hydroxide polymaltose complex are 3-4 times costlier than other iron preparations. Survey of iron formulations available in the market reveals that the cost of solid oral preparations with iron and folic acid ranges from Rs 0.15 to Rs 184.50(1230 fold difference). Comparison of medium cost of different iron formulations containing iron with folic acid shows that with drugs like ferrous gluconate, ferrous ascorbate, ferric hydroxide, iron polysucrose, the cost ranges from Rs 8 to 51.75 where as ferrous salts like ferrous sulphate, ferrous fumerate, ranges from Rs 1.25 to 5.00. Ferrous salts are cheap and have high content of elemental iron. They are better absorbed than ferric salts particularly at higher doses.

\section{Interaction between iron and food}

Dietary factors that influence absorption of iron can be classified into two types: ${ }^{15-17}$

\section{a. Absorption Inhibitors.}

\section{b. Absorption Enhancers.}

These affect the bioavailability of iron supplement into a great extent and hence require more attention.

\section{Absorption inhibitors}

Plant based diets contain phytate (myo-inositol hexakisphosphate) which is the main inhibitor of iron absorption. The effect of phytate on iron absorption is dose dependent and starts at very low concentrations i.e $2-10 \mathrm{mg} / \mathrm{meal}$. The ratio of phytate to iron can be used to determine the effect on absorption. If the ratio is $1: 1$ or $0.4: 1$ significantly improves absorption of iron in plain cereal or legume-based meals that do not contain any enhancers of iron absorption or $6: 1$ in composite meals with certain vegetables containing ascorbic acid and meat as enhancers. Poly phenols are present in various quantities in plant-based foods and beverages, for eg: vegetables, fruits, legumes, cereals, tea, coffee, and wine. The inhibiting effect of poly phenols on iron absorption is observed with black tea and to a lesser extent with herbal teas. Polyphenols in cereals and legumes show inhibitory effect of phytate. Calcium shows negative effects on haem and nonhaem iron absorption, which makes it different from other inhibitors that effect non-haem iron absorption only. Dose dependent inhibitory effects were observed at doses of 75-300mg when calcium was added to bread and at doses of $165 \mathrm{mg}$ calcium from milk products. Animal proteins like milk proteins, egg proteins, and albumin show inhibition of iron absorption. The two major milk proteins casein and whey and egg white show inhibitory effect of iron absorption. Proteins from soya bean decrease iron absorption. Presence of heavy metals like lead, cobalt, manganese, zinc and arsenic in food or in supplements can obstruct iron absorption. 


\section{Absorption enhancers}

The dose dependent enhancing effect of natural or added ascorbic acid on iron absorption has been explained by researchers. The enhancing effect of ascorbic acid is due to the ability to reduce poorly soluble ferric to soluble ferrous iron and also due to its potential to chelate iron. Ascorbic acid overcome the negative effect of iron absorption of all inhibitors(phytate, polyphenols, calcium, proteins in milk products)but increase the absorption of both natural and supplement iron. In fruits and vegetables, the enhancing effect of ascorbic acid is nullified by the inhibiting effect of polyphenols. Ascorbic acid is the only absorption enhancer in vegetarian diets and iron absorption from vegetarian meals can be increased by inclusion of ascorbic acid containing vegetables or fruits. Cooking, chemical processing and storage degrade ascorbic acid and decrease or remove its enhancing effect of iron absorption. The enhancing effect of meat, fish, and chicken on non-heam iron absorption from vegetarian meals has been shown and $30 \mathrm{gms}$ of meat is considered equivalent to $25 \mathrm{mg}$ of ascorbic acid. Addition of meat, fish or beef to a maize meal increases non-heam iron absorption 2-3 times.

\section{Role of pharmacist in the treatment of Anemia}

Considering all the above facts about the iron supplements and their possible bioavailability and cost factors, community pharmacist can educate the consumers about the selection of more efficient supplements. He can advise about the right way of administration, Food-Food interaction, Food-drug interaction that influence the absorption of iron. ${ }^{18-20}$ The community pharmacist should educate the anaemic patients ((who take iron supplements) by the following ways:

\section{Right's (Do's)}

a) Motivate to take immediate release iron supplements. Use combined therapy with ascorbic acid and folic acid.

b) Prefer the iron supplements containing divalent salts like ferrous sulphate, ferrous fumerate, ferrous succinate which are cheaper and more bioavailable.

c) Take the formulation on empty stomach. If it leads to nausea, gastritis or GI discomfort, then take the product 2 hours after the meals.

d) Make sure that the supplement contains not less than33mg of elemental iron.

e) Take the dosage form at least for 3 months on daily basis, if too many side effects are faced then take thrice a week for longer time.

f) Take the supplements with any juice (orange, lemon, grapes etc) that is rich in ascorbic acid which will help in improving bioavailability of iron.

\section{Wrong's (Don'ts)}

a. Don't take supplements containing wrong combinations like the ones with calcium, cobalt, zinc, amino acids and vitamin B complex.

b. Do not take eggs, milk products, soya with iron supplements because they decrease or inhibit the iron absorption.

c. Don't take calcium supplements with iron preparations. This is most common mistake done by pregnant women who are generally prescribed with these supplements.

d. Do not take iron supplements with food substances containing pulses, legumes, cereals which contain high amount of phytates that inhibits absorption of iron strongly.

e. Don't take fruits like apple, papaya and vegetables like spinach (rich in polyphenols\& phytates) along with iron preparations.

f. Don't take iron preparations with coffee, tea or wine (due to presence of polyphenols, phytates) which inhibit iron absorption.

\section{Conclusion}

Anaemia is a disease occurs due to iron deficiency (lack of $\mathrm{RBC}$ cells) and effecting millions of people around the world. Iron supplements which are taken orally are cheap, simple to use, safe and effective for treating anaemia. The therapy for anaemia includes right dosage form, right drug, right dose, right time, right combinations if any and most important one is right way and right time of administration to avoid inhibitory food-food \& drug-food interactions. As a community pharmacist, with depth knowledge and experience, he/she should guide the patients in a proper manner and help the Community \& Nation to make free anaemic India.

\section{Acknowledgements}

None.

\section{Conflict of interest}

The author declares that there is no conflict of interest.

\section{References}

1. Harsh Mohan. Text book of pathology. 5th ed. Hyderabad: Jaypee publications; 2005. 355-441 p.

2. Hunt JR. Bioavailability of iron, zinc, and other trace minerals from vegetarian diets. Am J Clin Nutr. 2003;78(3 Suppl):633S-639S.

3. Ines E, Hurrell R. Iron bioavailability and dietary reference values. Am J Clin Nutr. 2010;91(5):1461S-1467S.

4. Karelia BN, Buch JG. Analysis of hematinic formulations available in the Indian market. J Pharmacol Pharmacother. 2012;3(1):35-38.

5. $19^{\text {th }}$ WHO model list of Essential Medicines. Geneva: World Health Organization; $2015.53 \mathrm{p}$

6. Santiago P. The Scientific World Journal. 2011;10:1-5.

7. Heinrich HC. Bioavailability of trivalent iron in oral iron preparations. Therapeutic efficacy and iron absorption from simple ferric compounds and high- or low-molecular weight ferric hydroxide-carbohydrate complexes. Arzneimittelforschung. 1975;25(3):420-6.

8. Harington M, Holz C, Zeder C. A comparison of the bioavailability of ferrous fumarate and ferrous sulfate in non-anemic Mexican women and children consuming a sweetened maize and milk drink. Eur J Clin Nutr. 2011;65(1):20-25.

9. Gomes da Costa A, Vargas S, Clode N, et al. Prevalence and risk factors for iron deficiency anemia and iron depletion during pregnancy: A prospective study. Acta Med Port. 2016;29(9):514-518.

10. Sharma P, Mehta S, Nagar R. Prevalence of anemia and sociodemographic factors associated with anemia among pregnant women attending antenatal Hospital in Jaipur City, India. IOSR J Pharm Biol Sci. 2013;6(3):1-5. 
11. Bentley ME, Griffiths PL. The burden of anemia among women in India. Eur J Clin Nutr. 2003;57(1):52-60.

12. Haemoglobin Concentrations for the Diagnosis of Anaemia and Assessment of Severity. Geneva: World Health Organization; 2011.

13. Wang QM, Zhang SK, Hu M, et al. Establishment of quality assurance system of the National Free Preconception Health Examination Project in China. Chin Med J. 2015;95:166-168.

14. Le CH. The prevalence of anemia and moderate-severe anemia in the US population (NHANES 2003-2012). PLoS ONE. 2016;11(11):e0166635.

15. Behboudi-Gandevani S, Imani S, Moghaddam-Banaem L, et al. Can intrauterine contraceptive devices lead to VulvoVaginal Candidiasis (VVC) and Anemia in Iranian new users? Sex Reprod Healthc. 2015;6(1):40-43.

16. National Health and Family Planning Commission of the PRC. Report of investigation on the contraception effect of intrauterine device by one hundred and twenty thousand cases. Chin J Family Plan. 2007;15:132136.
17. Borghei A, Qorbani M, Borghei, et al. Effects of IUD on iron status in IUD users in Gorgan Iran. Med J Islam Repub Iran. 2011;25(3):131-135.

18. Blanco-Rojo R, Toxqui L, Lopez-Parra AM, et al. Influence of diet, menstruation and genetic factors on iron status: A cross-sectional study in Spanish women of childbearing age. Int J Mol Sci. 2014;15(3):40774087.

19. The Global Prevalence of Anaemia in 2011. Geneva: World Health Organization; $2015.48 \mathrm{p}$

20. Adamu AL, Crampin A, Kayuni N, et al. Prevalence and risk factors for anemia severity and type in Malawian men and women: Urban and rural differences. Popul Health Metr. 2017;15(1):12. 